\title{
Infrared Digital Focal Plane Arrays for Earth Remote Sensing Instruments ${ }^{+}$
}

\author{
Sarath Gunapala 1,*, Sir Rafol 1, David Ting 1, Alexander Soibel 1, Arezou Khoshakhlagh 1, \\ Sam Keo ${ }^{1}$, Brian Pepper ${ }^{1}$, Anita Fisher ${ }^{1}$, Cory Hill 1, Edward Luong 1, Kwong-Kit Choi ${ }^{2}$, \\ Arvind D'Souza ${ }^{3}$, Christopher Masterjohn ${ }^{3}$, Sachidananda Babu ${ }^{4}$ and Parminder Ghuman ${ }^{4}$ \\ 1 Center for Infrared Photodetectors, Jet Propulsion Laboratory, California Institute of Technology, \\ Pasadena, CA 91109, USA; sir.b.rafol@jpl.nasa.gov (S.R.); David.z.ting@jpl.nasa.gov (D.T.); \\ alexander.soibel@jpl.nasa.gov (A.S.); arezou.khoshakhlagh@jpl.nasa.gov (A.K.); \\ sam.a.keo@jpl.nasa.gov (S.K.); brian.j.pepper@jpl.nasa.gov (B.P.); anita.m.fisher@jpl.nasa.gov (A.F.); \\ cory.j.hill@jpl.nasa.gov (C.H.); edward.m.luong@jpl.nasa.gov (E.L.) \\ 2 NASA Goddard Space Flight Center, Greenbelt, MD 20771, USA; KNYCHOI@msn.com \\ 3 DRS Network \& Imaging Systems, Inc., Cypress, CA 90630, USA; Arvind.Dsouza@drs.com (A.D.); \\ christopher.masterjohn@drs.com (C.M.) \\ 4 NASA Earth Science Technology Office, Greenbelt, MD 20771, USA; sachidananda.r.babu@nasa.gov (S.B.); \\ p.ghuman@nasa.gov (P.G.) \\ * Correspondence: sarath.d.gunapala@jpl.nasa.gov; Tel.: +1-818-354-1880 \\ + Presented at the 15th International Workshop on Advanced Infrared Technology and Applications \\ (AITA 2019), Florence, Italy, 17-19 September 2019.
}

Published: 24 December 2019

\begin{abstract}
In this presentation, we will discuss the advantages of using an in-pixel digital read out integrated circuit and type-II strained layer superlattice detector array technology to elevate the operating temperature of the focal plane array for Earth remote sensing instruments.
\end{abstract}

Keywords: superlattice; infrared; detector; digital; focal plane array

\section{Introduction}

Long-Wavelength Infrared (LWIR) Focal Plane Arrays (FPAs) needed for Earth Science imaging, spectral imaging, and sounding applications have always been among the most challenging in infrared photodetector technology due to the rigorous material growth, device design and fabrication demands. Future small satellite missions will present even more challenges for LWIR FPAs, as operating temperature must be increased so that cooler (and radiator) volume, mass, and power can be reduced. To address this critical need, we are working on following two technologies. 1) Type-II Superlattice (T2SL) Barrier Infrared Detector (BIRD), which combines the high pixel-to-pixel operability, spatial uniformity, temporal stability, scalability, producibility, and affordability advantages of the Quantum Well Infrared Photodetector (QWIP) FPA with the better quantum efficiency and dark current characteristics. A Mid-Wavelength Infrared (MWIR) T2SL BIRD FPA is a key demonstration technology in the (6U) CubeSat Infrared Atmospheric Sounder (CIRAS) funded under the ESTO In-space Validation of Earth Science Technologies (InVEST) Program. 2) High dynamic range 3D Read Out Integrated Circuit (3D-ROIC), which integrates a digital reset counter with a conventional analog ROIC to provide a much higher effective well capacity than previously achievable. The resulting longer integration times are especially beneficial for high flux/dark current LWIR applications as they can improve signal-to-noise ratio and/or increase the operating temperature. By combining the aforementioned technologies, this project seeks to demonstrate a costeffective, high-performance LWIR FPA technology with significantly higher operating temperature and sensitivity than previously attainable, and with the flexibility to meet a variety of Earth Science 
thermal infrared (TIR) measurement needs, particularly the special requirements of small satellite missions.

The recent emergence of BIRDs such as the $\mathrm{nBn}$ [1] and the XBn [1] have resulted in MWIR and LWIR detectors with substantially higher operating temperatures than previously available in III-V semiconductor based MWIR and LWIR detectors. The initial nBn devices used either InAs absorber grown on InAs substrate, or lattice-matched InAsSb alloy grown on GaSb substrate, with fixed cutoff wavelengths of $\sim 3.2 \mu \mathrm{m}$ and $\sim 4 \mu \mathrm{m}$, respectively. While these detectors could operate at much higher temperatures than existing MWIR detectors based on InSb, their spectral responses do not cover the full $(3-5.5 \mu \mathrm{m})$ MWIR atmospheric transmission window.

\section{Barrier Infrared Detectors}

Much has been discussed in the literature about the barrier infrared detector [1], and unipolar barriers in recent years. The ideal $\mathrm{nBn}$ structure would have two n-type region (n) separated by a larger bandgap, undoped barrier layer (B), where the n-B heterojunctions have a larger conduction band offsets and zero valence band offsets. Such a barrier would block majority carrier electrons, but pass photogenerated holes. The $\mathrm{nBn}$ infrared detector is designed to reduce dark current (noise) without impeding photocurrent (signal). Central to the $\mathrm{nBn}$ operation is the strong suppression of generation-recombination (G-R) dark current due to Shockley-Read-Hall (SRH) processes. Another important aspect of $\mathrm{nBn}$ and related structures is their effectiveness in reducing surface leakage current.

The InAs/InAsSb (Gallium-free) T2SLS has emerged as an alternative adjustable bandgap, broad-band III-V IR detector material to the more established InAs/GaSb T2SL. Recently, there has been growing interest in this material as an infrared detector absorber due to longer MWIR and LWIR minority carrier lifetimes in InAs/InAsSb strained-layer superlattice (SLS) than in InAs/GaSb Superlattice (SL) and demonstrated an InAs/InAsSb SLS LWIR photodetector based on the $\mathrm{nBn}$ device design. The T2SLS material can be grown on InAs or GaSb substrates, GaSb is available in 2", $3 ", 4$ " and 6" diameters formats.

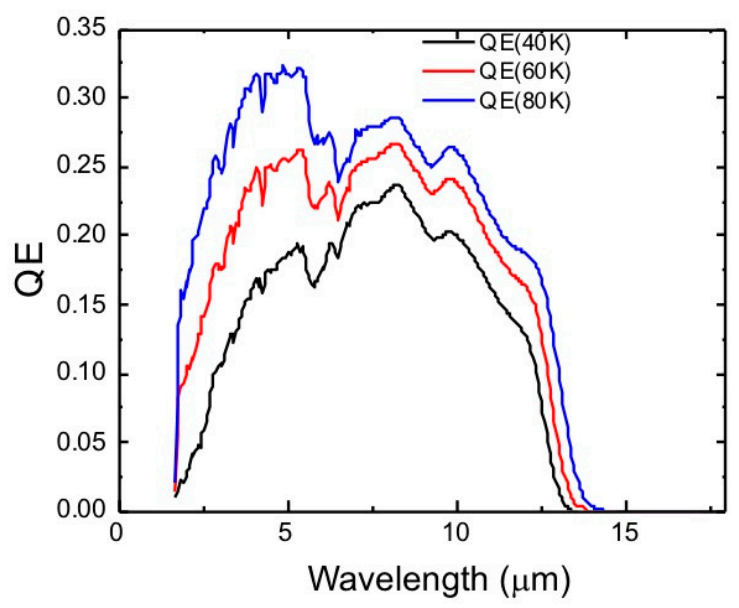

Figure 1. Backside illuminated spectral quantum efficiency (QE) for an LWIR detector measured at temperatures ranging from $40 \mathrm{~K}$ to $80 \mathrm{~K}$.

\section{LWIR BIRD Focal Plane Array}

An InAs/InAsSb SLS nBn structure was grown on a 4-inch diameter low Te-doped GaSb (100) substrate in a Veeco Applied-Epi Gen III molecular beam epitaxy machine. Square mesa photodiodes of area $250 \mu \mathrm{m} \times 250 \mu \mathrm{m}$ were fabricated along with detector arrays for responsivity and dark current measurements. The devices were not passivated nor treated with anti-reflection coating. Figure 1 shows the spectral QE derived from back-side illuminated (through the GaSb substrate) spectral responsivity measured at temperatures ranging from $40 \mathrm{~K}$ to $80 \mathrm{~K}$; the QE has not been corrected for 
substrate reflection or transmission. Accordingly, the spectral $\mathrm{QE}$ for $40 \mathrm{~K}$ and $80 \mathrm{~K}$ was taken at -50 $\mathrm{mV}$. As shown on Figure 1 the cut off wavelength (i.e., $\mathrm{QE}$ is $50 \%$ ) of the $\mathrm{QE}$ spectrum is $12.5 \mu \mathrm{m}$. The dark current density at $60 \mathrm{~K}$ is $2.6 \times 10^{-5} \mathrm{~A} / \mathrm{cm}^{2}$ at the operating bias of $-50 \mathrm{mV}$. The detector material was used to fabricate $24 \mu \mathrm{m}$ pitch, $640 \times 512$ format arrays and hybridized to the SBF-193 ROIC. The detector mesas are etched to just below the barrier; the pixels are not fully reticulated.

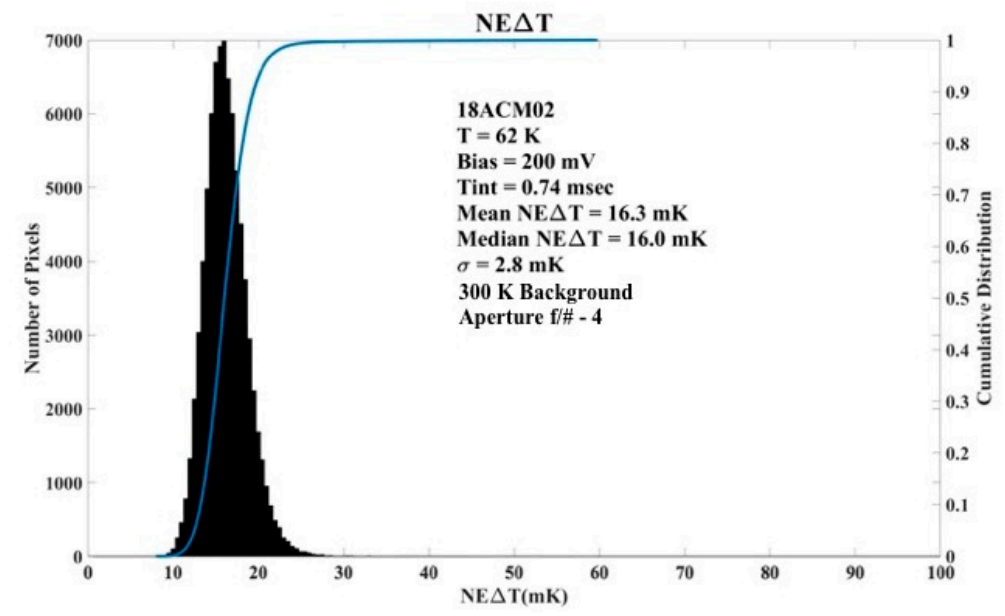

Figure 2. Noise equivalent temperature difference (NE $\Delta \mathrm{T}$ ) of the $12.5 \mu \mathrm{m}$ cutoff BIRD FPA at $62 \mathrm{~K}$.

The Noise Equivalent Temperature Difference (NE $\Delta \mathrm{T}$ ) provides the thermal sensitivity of an infrared imaging system and it is defined as the minimum temperature difference required at the target to produce unity signal-to-noise-ratio. The experimentally measured NE $\Delta \mathrm{T}$ of $16.3 \mathrm{mK}$ (Figure 2) is in fair agreement with the estimated NE $\Delta \mathrm{T}$ value based on the results of a single element test detector data.

\section{Digital Read Out Integrated Circuit}

Conventional ROICs are based on analog electronic circuits. The modern ROICs are Digital ROICs (DROICs). Conventional analog ROICs store charges at individual ROIC pixels and route them out via output taps to off-chip analog-to-digital converters (ADCs) or route them to on-chip column parallel ADCs. This method requires a very large ROIC in-pixel well depth to achieve high Signal-to-Noise-Ratio (SNR). In DROICs the charges get digitized at ROIC individual pixel level with a counter by incrementing each time a small charge bucket gets filled. Ideally, this could provide a very high effective well depth for DROIC pixels compared to conventional analog ROIC pixels. Total well depth of DROIC pixel is given by the size of the charge bucket times the number of counts of the in-pixel counter [2].

The ultimate sensitivity (i.e., highest SNR) of an infrared FPA is determined by the maximum well depth of the ROIC pixels assuming the total noise of the FPA is determined by the shot noise (i.e., statistical fluctuations of the signal) of the photocurrent. Therefore, a DROIC with higher bit counter could provide a higher SNR or could operate a DFPA at higher operating temperature with same SNR as a conventional FPA (i.e., same detector array with an analog ROIC).

Figure 3 shows a case study performed with the experimental values obtained from the $12.5 \mu \mathrm{m}$ cutoff LWIR BIRD FPA operating at various temperatures from $60 \mathrm{~K}$ to $110 \mathrm{~K}$ with $250 \mathrm{~K}$ background and $\mathrm{f} / 1.4$ aperture. Figure 3 clearly shows the two LWIR Landsat bands, from 10.3-11.3 $\mu \mathrm{m}$ and 11.5$12.5 \mu \mathrm{m}$ (Landsat bands) can achieve $20 \mathrm{mK}$ NE $\Delta \mathrm{T}$ with a DROIC with 100 million electrons well depth. Furthermore, LWIR BIRD FPAs could operate at even higher temperature with DRIOCs having greater effective well depths. 


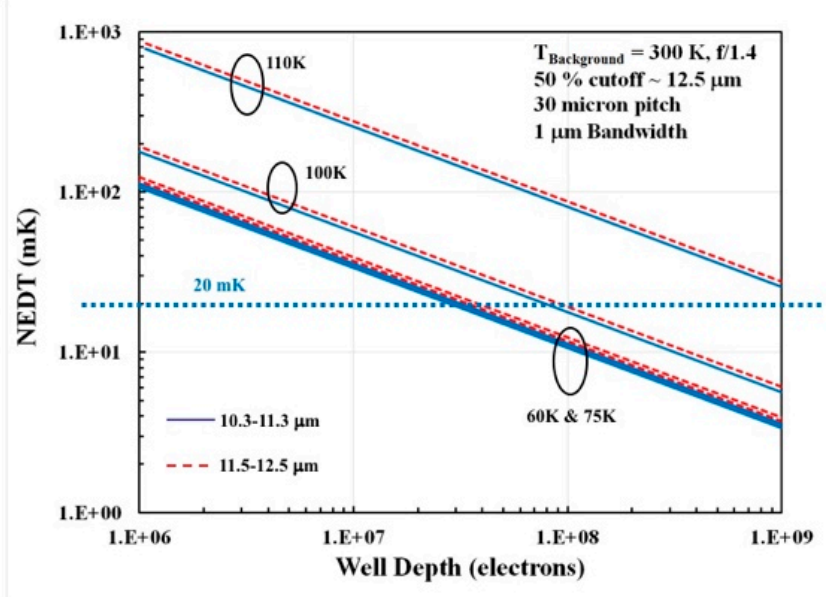

Figure 3. NE $\Delta$ T vs. ROIC well depth as a function of LWIR BIRD FPA operating temperature for two Landsat wavelength bands (from 10.3-11.3 $\mu \mathrm{m}$ and 11.5-12.5 $\mu \mathrm{m}$ ) with $250 \mathrm{~K}$ background and $\mathrm{f} / 1.4$ aperture.

\section{Summary}

Ga-free T2SLS LWIR BIRD FPA could easily operates at 20K higher operating temperature (HOT) (i.e., compared to QWIP FPA) due to the strong suppression of G-R dark current due to SRH processes as explained earlier. Another 20K higher operating temperature advantage can be achieved when we further improve the performance by hybridizing the T2SLS LWIR BIRD detector array to the high-dynamic range DROIC from DRS. Therefore, a T2SLS LWIR BIRD DFPA can easily operates at much higher operating temperature compared to a conventional LWIR FPA.

Author Contributions: Conceptualization, S.G.; methodology, D.T; validation, A.S; formal analysis, S.R; investigation, A.K, S.K., B.P., A.F., E.L.; resources, P.G. and S.B.; data curation, K.-K.C., A.D., and C.M.; writingreview and editing, S.G.; visualization, C.H.; project administration, P.G. and S.B.

Funding: This research was funded by NASA Earth Science Technology Office.

Acknowledgments: The authors thank Nikzad Toomarin, Harish Manohara, Carl Ruoff, Eastwood Im, Randy Odle, and Jason Hyon of Jet Propulsion Laboratory for encouragement and support. The research described in this paper was carried out at the Jet Propulsion Laboratory, California Institute of Technology, under a contract with the National Aeronautics and Space Administration. @ 2019. All rights reserved. Government sponsorship acknowledged.

\section{References}

1. Ting, D.Z.; Rafol, S.B.; Keo, S.A.; Nguyen, J.; Koshakkhlagh, A.; Soibel, A.; Hoglund, L.; Fisher, A.M.; Luong, E.M.; Mumolo, J.M.; et al. InAs/InAsSb Type-II Superlattice Mid-Wavelength Infrared Focal Plane Array With Significantly Higher Operating Temperature Than InSb. IEEE Photonics J. 2018, 10, 1-6.

2. Schultz, K.I.; Kelly, M.W.; Baker, J.J.; Blackwell, M.H.; Brown, M.G.; Colonero, C.B.; David, C.L.; Tyrrell, B.M.; Wey, J.R. Digital-Pixel Focal Plane Array Technology. Linc. Lab. J. 2014, 20, 36-51.

(c) 2019 by the authors. Licensee MDPI, Basel, Switzerland. This article is an open access article distributed under the terms and conditions of the Creative Commons Attribution (CC BY) license (http://creativecommons.org/licenses/by/4.0/). 